\title{
Severe skin rash and liver toxic effects caused by first-line anti-tuberculosis drugs: a case report
}

\begin{abstract}
Adverse reactions caused by anti-tuberculosis drugs are the most common adverse reactions in the clinical practices of anti-tuberculosis treatments. A 56-year-old male patient was found with large areas of skin rash all over the body (especially at the skins of the chest, abdomen, and limbs) with pruritus at 2 weeks after oral intake of anti-tuberculosis drugs for the treatment of pleural tuberculosis. The patient was also found with the presentations of aversion to oil, nausea, vomiting, and yellowing of the sclera and the skin all over the body. Routine biochemical tests showed severe liver dysfunction. After hospitalized, antiallergic therapy and combined application of liver-protection drugs was performed for the symptomatic treatment. The liver function of the patient recovered gradually in half a month, the skin rashes disappeared, and the scurf fell off. The vital signs of the patient were stable now, and anti-tuberculosis treatment could be performed again after the related indexes were measured. We speculated that the adverse reactions in this patient could be associated with the age, the genotype of slow isoniazid acetylated metabolism, and the pyrazinamide which could easily cause liver toxicities in the anti-tuberculosis strategy used. The major measure in preventing adverse reactions in the anti-tuberculosis treatments is the dynamic liver function monitoring in the target population, which could help the early identification, early discontinue the drug therapy, and early treatment.
\end{abstract}

Keywords: severe skin rash, liver toxic effects, anti-tuberculosis drugs, case report
Volume 5 Issue 4 - 2017

\author{
Meiling Yu, Daohua Guo, Yongquan Hu, \\ Xiaoxiang Wu \\ Department of Pharmacy, Hospital of Bengbu Medical College, \\ China
}

Correspondence: Daohua Guo, Department of Pharmacy, the first Affiliated Hospital of Bengbu Medical College, Anhui, Bengbu, 233004, P.R. China, Tel 86552 3086236, Email2477160265@qq.com

Received: October 26, 2016 | Published: February 17, 2017

\section{Introduction}

The adverse reactions of anti-tuberculosis drugs mainly include drug-induced liver injury, gastrointestinal reactions, and skin rash. Currently, the first-line anti-tuberculosis strategy in China includes the application of isoniazide, rifampicin, pyrazinamide, and ethambutol. Despite the high effectiveness, the serious adverse reactions caused by this strategy may cause a lot of patients discontinue the treatment. ${ }^{1}$ The most common adverse reaction caused by the first-line antituberculosis drugs is mild liver dysfunction, ${ }^{2}$ while severe liver toxic effects including liver failure or even death are very rare. The other adverse reactions caused by the first-line anti-tuberculosis drugs include rashes on the local or full body skin, ${ }^{3}$ of which maculopapule is the most common type.

Previous studies have shown that the mechanisms involved in the liver damages caused by anti-tuberculosis drugs include the formation of active metabolites caused by impaired liver detoxification functions, activation of immune responses, slow acetylation, and re-activation of human herpes virus. ${ }^{4}$ For the patients with NAT2 gene polymorphism (slow acetylating) or underlying immune deficiencies that are with relatively high risk of liver toxic effects, no obvious clinical presentation could be found at the initial stage of the development and progression of the liver toxicity (for instance, mild liver damages could be only presented with slight increase of transaminase level), and the adverse reactions could only be found in the biochemical routine tests. The yellowing of the sclera and the skin all over the body and the occurrence of pruritus reflect that the patients are with relatively severe liver dysfunction (ALT $\geq 3$ times of the upper limit of the normal range), and drug therapy is required to prevent the development of liver failure or even death. These facts suggest that the liver function of the patients should be closely monitored during the anti-tuberculosis treatment.

Skin rash is the second leading adverse reaction caused by anti- tuberculosis drugs. Mild to moderate pruritus is often accompanied with the occurrence of skin rash, and allergic shock may even occur in severe cases. Thus the healthcare givers should be very careful, and the dose of anti-tuberculosis drugs should be reduced or even stopped according to the severity of the disease in the patients with skin rash. Severe liver toxicity and drug induced skin rash are important adverse reactions causing the discontinuation of anti-tuberculosis treatment. How to perform a new cycle of anti-tuberculosis treatment after the liver protection, which alleviated the liver toxicity and eliminated the skin rash is also a challenge for the clinicians.

\section{Case report}

A 56-year-old male patient admitted to our hospital for nausea, vomiting, and large areas of skin rash (at the face, abdomen, chest, and limbs) for 1 week, with progressive aggravation after oral intake of anti-tuberculosis drugs for the treatment of tuberculous pleuritic (Table 1). On admission, the physical examination revealed large areas of maculopapule with pruritus at the skin of the face, chest, abdomen, and limbs, while no obvious skin rupture was found (Figures $1 \mathrm{~A} \&$ B). Hair loss was noticed, but no obvious skin yellowing was found. Obvious yellowing of the sclera and moderate pleural effusion was found. The liver of the patient was enlarged, and could be touched at $10 \mathrm{~cm}$ under the xiphoid process with tenderness. The temperature of the patient was $38^{\circ} \mathrm{C}$, the pulse was 88 beats/min, respiration was 19 times/min, blood pressure was $120 / 88 \mathrm{mmHg}$, and heart rate was 88 beats/min. Eight index of immunity test showed no abnormality. Liver function examinations showed that the ALT level was >1000U/L, AST level was $101 \mathrm{U} / \mathrm{L}$, ALP level was $205 \mathrm{U} / \mathrm{L}$ (Table 2 ), Y-GGP level was $265 \mathrm{U} / \mathrm{L}$, albumin level was $31.6 \mathrm{~g} / \mathrm{L}$, albumin-globulin ratio was 1.01 , and prothrombin activity (PT- $\%$ ) was $59.9 \%$. These laboratory examination results suggested that the patient was with severe liver dysfunction. X-ray showed focal shadow at the bilateral pleura. The patient was initially diagnosed with tuberculous pleurisy, drug 
induced skin rash (the susceptible drug of anti-tuberculosis drug), and acute liver dysfunction (possible drug-induced liver injury, with the susceptible drug of anti-tuberculosis drug). After admission, all the anti-tuberculosis drugs were stopped (the patient had used isoniazide, pyrazinamide, and ethambutol), but the skin rash and liver damages were not improved. Glucocorticoids are generally used for the treatment of drug induced skin rash; however, the underlying disease of this patient was tuberculous pleurisy accompanied with moderate pleural effusion, and the toxic reactions caused by tuberculosis were evident, thus intravenous application of glucocorticoids could result in the diffusion of tuberculosis. Therefore, hydrocortisone butyrate ointment that containing less glucocorticoids was applied on the skin of the patient (twice/day) to treat the skin rash, along with the oral administration of levocetirizine $(5 \mathrm{mg}$, once/day) and intravenous injection of $10 \%$ calcium gluconate to alleviate the allergic symptoms of the skin. While for the liver dysfunction which occurred recently, reduced glutathione, hepatocyte growth-promoting factors, and oxymatrine sodium chloride solution were intravenously injected for the liver protection. The liver function of the patient recovered to almost normal (ALT $98 \mathrm{U} / \mathrm{L}$, AST $62 \mathrm{U} / \mathrm{L}$, total protein $68.3 \mathrm{~g} / \mathrm{L}$ ) at 4weeks after the administration (Table 2 ), the yellowing of the skin reduced, pruritus improved evidently, the colour of the rash skin darkened, and large areas of scurf fell off was found (Figure 1C \& D).

Table I Dosage and frequency of anti-tuberculosis drugs

\begin{tabular}{lll}
\hline Medication & Dosage & Dosing schedule \\
\hline Isoniazid(H) & $0.3 \mathrm{~g}$ & Once daily \\
Pyrazinamide(Z) & $\mathrm{I.5g}$ & Once daily \\
Ethambutol(E) & $\mathrm{Ig}$ & Once daily \\
\hline
\end{tabular}

Table 2 Changes of liver function in patients before and after treatmen

\begin{tabular}{|c|c|}
\hline Liver function at different time & Check value \\
\hline \multicolumn{2}{|l|}{ After Anti-tuberculosis treatment } \\
\hline $\mathrm{ALT}(\mathrm{U} / \mathrm{L})$ & 287 \\
\hline AST(U/L) & 205 \\
\hline $\mathrm{TBIL}(\mu \mathrm{mol} / \mathrm{L} \square$ & 235 \\
\hline$A L P(U / L)$ & 101 \\
\hline GGT(U/L) & $>1000$ \\
\hline \multicolumn{2}{|l|}{$\begin{array}{l}\text { After Hepatoprotetcive treatment } \\
\text { The first day after treatment }\end{array}$} \\
\hline $\begin{array}{l}\operatorname{ALT}(U / L) \\
\operatorname{AST}(U / L) \\
\operatorname{TBIL}(\mu \mathrm{mol} / L \square \\
\text { ALP(U/L) } \\
\text { GGT(U/L) }\end{array}$ & $\begin{array}{l}265 \\
185 \\
217 \\
464 \\
812\end{array}$ \\
\hline \multicolumn{2}{|l|}{ The fifth day after treatment } \\
\hline $\begin{array}{l}\mathrm{ALT}(\mathrm{U} / \mathrm{L}) \\
\mathrm{AST}(\mathrm{U} / \mathrm{L})\end{array}$ & 148 \\
\hline $\begin{array}{l}\text { AST(U/L) } \\
\text { TBIL(umol/L } \square\end{array}$ & 122 \\
\hline $\mathrm{ALP}(\mathrm{U} / \mathrm{L})$ & 201 \\
\hline $\mathrm{GGT}(\mathrm{U} / \mathrm{L})$ & 157 \\
\hline The tenth day after treatment & 444 \\
\hline $\mathrm{ALT}(\mathrm{U} / \mathrm{L})$ & 136 \\
\hline AST(U/L) & 117 \\
\hline TBIL $(\mu \mathrm{mol} / \mathrm{L} \square$ & 119.7 \\
\hline $\operatorname{ALP}(U / L)$ & 62 \\
\hline GGT(U/L) & 98 \\
\hline
\end{tabular}

A

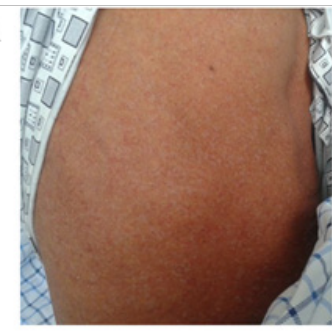

B
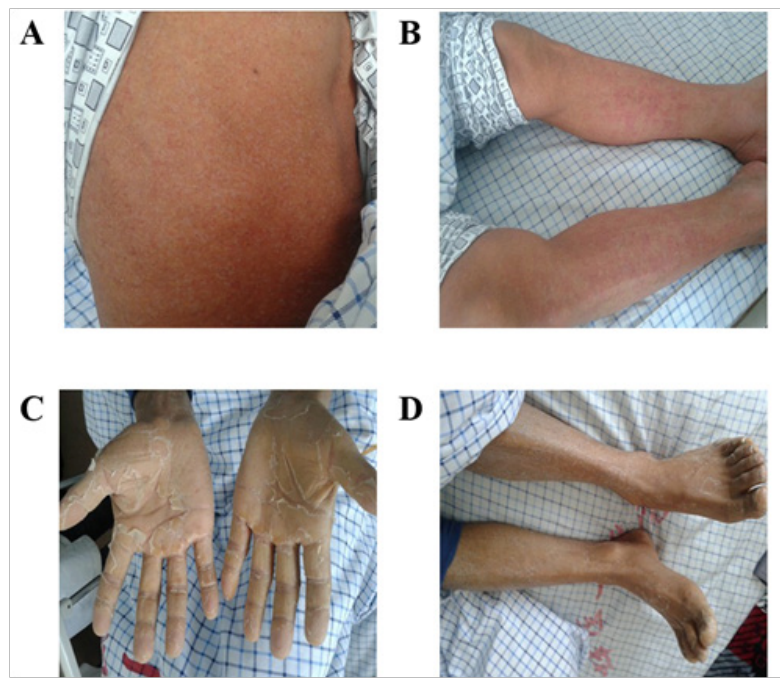

D

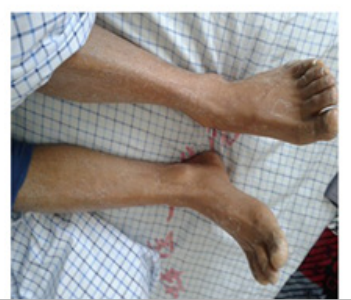

Figure I Generalized skin eruption and exfoliation showed in whole body on the first day of hospitalization (A, B). After treatment, the rash subsided and the skin was removed by large area $(C, D)$.

\section{Discussion}

Drug-induced liver injuries caused by anti-tuberculosis drugs are very common. In the present study, the patient was found with severe skin rash and liver dysfunction within 15 days after the intake of anti-tuberculosis drugs (including isoniazide, pyrazinamide, and ethambutol, while no alcohol drinking, alcoholic beverage drinking, or smoking was reported during the drug intake), which did not improve after the drugs stopped. The ALT of the patient was above 1000U/L on admission, which was higher than the upper limitation of detect of the biochemical test in our hospital; the highest AST level was $>464 \mathrm{U} / \mathrm{L}$, and even enzyme-jaundice separation was found. In addition, the patient was with large areas of skin rash all over the body, along with slight hair loss and severe epidermis exfoliation. Only very few cases with two severe adverse reactions have been reported to date.

Skin rash is very common during the application of antituberculosis drugs. Previous studies have already demonstrated that skin rash caused by first-line anti-tuberculosis drugs is associated with genetic variations of CYP2C19 and CYP2C9 genes. ${ }^{3,5} \mathrm{~A}$ recent study in Korea showed that tuberculosis patients with $\mathrm{ABCC} 2$ gene haplotype or polymorphism were with high risk of developing skin rash after the intake of first-line anti-tuberculosis drugs. ${ }^{6}$ As all the 3 anti-tuberculosis drugs the patient used could cause epidermis exfoliative dermatitis, it is still not clear which drug is the cause of the severe skin rash appeared in this patient.

Isoniazid is an essential component in the universally accepted first-line anti-tuberculosis strategies. The ALT level of the patients will increased to $\geq 5$ times of the upper limit of the normal range at 2-3months after the intake of isoniazid. Some patients are with some certain degree of adaptability, and the ALT level in them will decrease gradually after the drug stopped. However, the self-repair mechanism in some other patients could not be initiated due to the polymorphism of isoniazid metabolism-related $\mathrm{N}$-acetyltransferase, and thus liver dysfunction will occur. Thus isoniazid has been considered the major drug inducing liver toxicity in the first-line anti-tuberculosis drugs. The NAT2 in the liver of the patients could metabolize the isoniazid into acetylisoniazid, which could be oxidized by CYP4502E1 into liver toxic intermediate, damage the hepatocytes, and thus result in 
different degrees of liver toxicity. Previous studies have shown that NAT2 gene is highly polymorphism, which could change the function of NAT2. ${ }^{7}$ NAT2 acetylation gene polymorphism include fast, moderate, and slow metabolism types, while slow metabolism type could evidently induce the development of liver toxicity, which will affect the efficacies of anti-tuberculosis treatments and the occurrence of the adverse effects, especially when long-term administration of large dose of isoniazid was performed. ${ }^{8}$ The incidence of druginduced liver injury has been shown to be $51.2 \%$ in patients with slow acetylation genes after intake isoniazid, while the incidence in the ones with fast acetylation genes is only $25.2 \% .{ }^{9}$ Most of the Han population are with slow isoniazid acetylated metabolism genotypes including 191A, 481T, 590A, and 857A. ${ }^{10} \mathrm{~A}$ recent study showed that after the application of standard dose strategy (namely initial treatment strategy with the dose of isoniazid of $5 \mathrm{mg} / \mathrm{kg}$ ), $7 / 9$ of the patients with slow metabolism genotypes may suffer from isoniazid induced liver injury within 4weeks. ${ }^{11}$ The patient in the present case was found with liver injuries within 15 days after the intake of $5 \mathrm{mg} / \mathrm{kg}$ isoniazid (standard dose). Laboratory tests showed that both ALT and AST levels were over 5 times of the upper limits of the normal ranges. The AST and ALT levels decreased gradually to about 2 imes of the upper limits of the normal ranges after 10days of aggressive treatment. As the liver injury in the patient occurred so rapid and was so severe, and the injury lasted for such long time, we speculate that the patient may carrying a slow isoniazid acetylated metabolism genotype.

Pyrazinamide and ethambutol are two drugs commonly used in combination with isoniazid in the first-line anti-tuberculosis strategies. The adverse effect of ethambutol is mainly optic nerve damages, while liver injury is very rare. Pyrazinamide could cause arthralgia, appetite decrease, fever, and liver injury, ${ }^{12}$ as well as skin rash in some cases. Multi-center studies with large sample sizes in China have shown that ${ }^{13}$ pyrazinamide in the HRZ strategy is a relatively high risk factor for liver injury, and the liver toxicity is dependent on the dose of pyrazinamide. Continuous intake of pyrazinamide with the dose of $30 \mathrm{mg} / \mathrm{kg} / \mathrm{d}$ for 2 months is relatively safe, while the dose of $\geq 40 \mathrm{mg} / \mathrm{kg} / \mathrm{d}$ could cause liver injuries. ${ }^{14}$ The weight of the patient in the present case was $60 \mathrm{~kg}$, and the daily dose was $1.5 \mathrm{~g}$, which was evidently lower than the minimum toxic dose of the short-term administration of the drug, thus the risk of liver injury in short-term was very low. However, recent studies have shown that ${ }^{15}$ administrating pyrazinamide with the dose recommended in the Taiwan guideline is an independent risk factor of developing liver injury at 2 weeks after the drug intake. Thus pyrazinamide is considered as the drug that most easily induces liver toxicities in the first-line anti-tuberculosis drugs. Therefore, we also speculate that the severe liver toxicity in the present case could also be associated with the use of pyrazinamide.

\section{Conclusion}

In summary, within 2 weeks after the use of first-line antituberculosis strategy, the patient in the present case was with the presentations of liver failure including severe yellowing of the sclera and the skin all over the body, sharp increase of ALT and AST (ALT level above 200times of the upper limit of the normal range, and AST level above 10times of the upper limit of the normal range), and enzyme-jaundice separation. We speculated that the patient was with a high possibility of carrying slow isoniazid acetylated metabolism genotype; in addition, similar to isoniazid, the incidence of the liver toxicity induced by pyrazinamide is also very high, thus we speculated that the liver injury in the present case could be caused by the combined effects of pyrazinamide and isoniazid.
Currently, the most effective method to prevent the occurrence of severe cases is the dynamic monitoring of the liver function. However, no agreement in the frequency of the monitoring is available to date. One examination every one to two weeks should be considered if possible, especially for the patients with the ages of about 60years (previous studies have shown that for the patients over 40years old, the incidence and severity of adverse liver reactions are positively associated with the age ${ }^{16}$ Asians, and with many years history of drinking. When re-initiating the anti-tuberculosis treatment for the patient in the present case, rifampicin and/or ethambutol that with relatively low liver toxicity should be considered first when the ALT level recovered to lower than 2times of the higher upper limit of the normal range. If the liver function improves in the following 6-7days, the dose of isoniazid should be adjusted according to the metabolism genotypes of the patients if possible, and then the other anti-tuberculosis drugs should also be resumed to effectively manage the tuberculosis without inducing severe liver dysfunction, as well as further improve the adherence of the patients.

\section{Acknowledgments}

None.

\section{Conflicts of interest}

Author declares there are no conflicts of interest.

\section{Funding}

None.

\section{References}

1. Schaberg T, Rebhan K, Lode H. Risk factors for side-effects of isoniazid, rifampin and pyrazinamide in patients hospitalized for pulmonary tuberculosis. Eur Respir J. 1996;9(10):2026-2030.

2. Tostmann A, Boeree MJ, Aarnoutse RE, et al. Antituberculosis druginduced hepatotoxicity, concise up-to-date review. $J$ Gastroenterol Hepatol . 2008;23(2):192-202.

3. Kim SH, Kim SH, Yoon HJ, et al. GSTT1 and GSTM1 null mutations and adverse reactions induced by antituberculosis drugs in Koreans. Tuberculosis (Edinb). 2010;90(1):39-43.

4. Lee JY, Seol YJ, Shin DW, et al. A case of the drug reaction with Eosinophilia and Systemic Symptom (DRESS) Following Isoniazid Treatment. Tuberc Respir Dis (Seoul). 2015;78(1):27-30.

5. Kim SH, Kim SH, Yoon HJ, et al. CTP2C9, CYP2C19 and CYP2E1 genetic polymorphisms in anti-TB drug-induced maculopapular eruption. Eur J Clin Pharmacol. 2011;67(2):121-127.

6. SH Kim, YK Jee, JH Lee, et al. ABCC2 haplotype is associated with abtituberculosis drug-induced maculopapular eruption. Allergy Asthma Immunol Res. 2011;4(6):362-366.

7. Teixeira RL, Morato RG, Cabello PH, et al. Genetic polymorphisms of NAT2, CYP2E1 and GST enzymes and the occurrence of antituberculosis drug-induced hepatitis in brazilian TB patient. Mem Inst Oswaldo Cruz. 2011;106(6):716-724

8. Santos NP, Callegari-Jacques SM, Ribeiro Dos Santos AK, et al $\mathrm{N}$-acetyl transferase 2 and cytochrome P450 2E1 genes and isoniazidinduced hepatotoxicity in Brazilian patients. Int $J$ Tuberc Lung Dis. 2013;17(6):499-504

9. Bose PD, Sarma MP, Medhi S. Role of polymorphic N-acetyl transferase2 and cytochrome P4502E1 gene in antituberculosis treatment-induced hepatitis. J Gastroenterol Hepatol. 2011;26(2):312-318. 
10. Lee SW, Wu, L. SH, et al. NAT2 and CYP2E1 polymorphisms and susceptibility to first-line anti-tuberculosis drug-induced hepatitis. Int $J$ Tuberc Lung Dis. 2010;14(5):622-626.

11. Lin HJ, Han CY, Lin BK, et al. Ethnic distribution of slow acetylator mutation in the polymorphic N-acetyItransferase(NAT2) gene. Pharmacogenetics. 1994;4(3):125-134.

12. Azuma J, Ohno M, Kubota R, et al. NAT2 genotype guided regimen reduces isoniazid-induced liver injury and early treatment failure in the 6-month four-drug standard treatment of tuberculosis: a randomized controlled trial for pharmacogenetics-based therapy. Eur J Clin Pharmacol. 2013;69(5):1091-1101.

13. An $H$, Wu X, Wang $Z$. The clinical characteristics of anti-tuberculosis drug induced liver injury in 2457 hospitalized patients with tuberculosis in China. AFR J Pharmacol. 2013;7(13):710-714.
14. Pasipanodya JG, Gumbo T. Clinical and toxicodynamic evidence that high-dose pyrazinamide is not more hepatotoxic than the low doses currently used. Antimicrob Agents Chemother. 2010;54(7):2847-54.

15. Shu CC, Lee CH, Lee MC, et al. Hepatotoxicity due to first-line antituberculosis drugs: a five-year experience in a Taiwan medical centre. Int J Tuberc Lung Dis. 2013;17(7):934-939.

16. Abbasi MA, Ahmed N, Suleman A, et al. Common risk factors for the development of anti tuberculosis treatment induced hepatotoxicity. $J$ Ayub Med Coll Abbottabad. 2014;26(3):384-388. 